Article

\title{
Genome-Wide Identification and Comparative Analysis of MYB Transcription Factor Family in Musa acuminata and Musa balbisiana
}

\author{
Lin Tan ${ }^{1,+}$, Usman Ijaz ${ }^{2, \dagger}$, Haron Salih ${ }^{1}$, Zhihao Cheng ${ }^{1}$, Nwe Ni Win Htet ${ }^{1}$, Yu Ge ${ }^{1(\mathbb{C}}$ \\ and Farrukh Azeem 1,2,*(D) \\ 1 Haikou Experimental Station, Chinese Academy of Tropical Agricultural Sciences (CATAS)-Hainan Key \\ Laboratory of Banana Genetic Improvement, Haikou 571101, Hainan, China; tanlin@catas.cn (L.T.); \\ salih234@yahoo.com (H.S.); zhihaocheng1@catas.cn (Z.C.); \\ nweniwinhtet@gmail.com (N.N.W.H.); geyu@catas.cn (Y.G.) \\ 2 Department of Bioinformatics and Biotechnology, Government College University, \\ Faisalabad 38000, Pakistan; usmanijazahmad1246@gmail.com \\ * Correspondence: azeuaf@hotmail.com \\ + These authors contributed equally to this work.
}

Received: 21 January 2020; Accepted: 21 March 2020; Published: 27 March 2020

\begin{abstract}
MYB transcription factors (TFs) make up one of the most important TF families in plants. These proteins play crucial roles in processes related to development, metabolism, and stimulus-response; however, very few studies have been reported for the characterization of MYB TFs from banana. The current study identified 305 and 251 MYB genes from Musa acuminata and Musa balbisiana, respectively. Comprehensive details of MYBs are reported in terms of gene structure, protein domain, chromosomal localization, phylogeny, and expression patterns. Based on the exon-intron arrangement, these genes were classified into 12 gene models. Phylogenetic analysis of MYBs involving both species of banana, Oryza sativa, and Arabidopsis thaliana distributed these genes into 27 subfamilies. This highlighted not only the conservation, but also the gain/loss of MYBs in banana. Such genes are important candidates for future functional investigations. The MYB genes in both species exhibited a random distribution on chromosomes with variable densities. Estimation of gene duplication events revealed that segmental duplications represented the major factor behind MYB gene family expansion in banana. Expression profiles of MYB genes were also explored for their potential involvement in acetylene response or development. Collectively, the current comprehensive analysis of MYB genes in both species of banana will facilitate future functional studies.
\end{abstract}

Keywords: MYB transcription factor; expression profile; Musa acuminata; M. balbisiana; ripening

\section{Introduction}

Transcription factors (TFs) perform an essential role in the regulation of gene expression by suppressing or activating their target genes to control various phases of plant development and growth [1]. MYB TFs are commonly present in fungi, vertebrates, and plants, and make up one of the biggest TF families in plants [2]. The members of the MYB family contain a highly conserved and distinctive N-terminal DNA-binding or protein-protein interaction domain that typically bears one to four imperfect repeats of a particular sequence (termed R1, R2, R3, and R4) and three $\alpha$-helices. Each repeat comprises almost 52 amino acids in length [3]. The second and third helices form a special structure called a helix-turn-helix $(\mathrm{HTH})$ that binds to the major grooves of DNA [4,5]. However, the $C$ terminal region of the MYB domain is greatly divergent. It acts as an activation domain for the wide-ranging roles of the MYB family [6]. The number of repeats in the MYB domain is used as a 
basic criterion to classify MYB proteins. In plants, the MYB family is subdivided into four subfamilies, namely, "MYB-related proteins" (1R-MYB), "R2R3-MYB proteins" (2R-MYB), "R1R2R3-MYB proteins" (3R-MYB), and " $4 \mathrm{R}$ or Atypical MYB protein" (4R-MYB). In higher plants, the MYB members with two repeats (R2R3) are predominantly present [7].

Since the identification of the first MYB gene in plants, research has been broadly conducted in plants to identify and functionally characterize other members of this gene family $[2,8]$. Numerous MYB proteins are important for the control of several biochemical and physiological processes of plant development, primary/secondary metabolism, and plant response to abiotic and biotic stresses $[2,9,10]$ (Table S1). For example, the betalain pathway is regulated by BvMYB1 in beets [11]. Several studies have shown that MYB TFs are involved in the activation or repression of genes associated with the production of pigments/anthocyanins in fruits [12]. Furthermore, MYB TFs (FaEOBII and FaMYB10) also initiate the production of volatile compounds during the ripening of strawberry fruit [13]. The conical cell shape development of the epidermal cells is regulated by DhMYB1 in the flower labellum of Dendrobium hybrida [14]. In cotton, MYB108 is involved in response to Verticillium dahliae infection [15]. Moreover, there are several other examples of potential MYB gene involvement in some characteristic traits of plants, including wine-quality formation in grapes, legume-specific nodulations, and pollinator preferences $[16,17]$.

Banana (Musa acuminata L.) is an herbaceous plant from monocotyledons that is generally distributed in tropical and subtropical regions. The two species of Musa (M. acuminata with an AA genome Musa balbisiana with a BB genome) belong to the Musaceae family $(2 n=22$ chromosomes) and represent the two key ancestors of cultivated varieties of banana $[18,19]$. Despite its role in plant growth, development, cell differentiation, fruit ripening, and stress response, there is an absolute dearth of research focusing on MYB genes in banana plants $[18,20,21]$. Whole-genome sequencing of M. acuminata and M. balbisiana has facilitated study of genome-wide evolution and divergence of the gene families in these plants. The present study systematically identified $M Y B$ genes by evaluating the sequences of two Musa genomes (M. acuminata and M. balbisiana) using a set of bioinformatics tools. The results of this study will give insight into the organization, distribution, and evolution of the MYB gene family. Moreover, the expression pattern of MYB genes will contribute to functional characterization and understanding the biological role of these genes in banana.

\section{Results and Discussion}

\subsection{In Silico Identification and Sequence Characterization of MYB Family Genes}

The MYB domain sequences of Oryza sativa and Arabidopsis thaliana were employed as a query for the identification of MYB proteins present in the genomes of M. acuminata and M. balbisiana. The redundant sequences and candidate genes with imperfect open reading frames (ORFs) were discarded and remaining sequences were used for further analysis. For additional confirmation of the MYB domain in identified proteins, the sequences were subjected to Pfam and PROSITE screening. After carefully surveying the M. acuminata and M. balbisiana MYB proteins and confirming the conserved characteristics of motifs and domains, 305 non-redundant MYBs were predicted in M. acuminata and 251 in M. balbisiana. These genes were termed as MaMYB and MbMYB (Table S2). The MYB members have been studied in diverse plant species (Table 1). Given the genome size in both Musa spp., the number of $M Y B$ proteins is expected to be two to three times larger than $A$. thaliana and similar to O. sativa. However, the comparison of genome size and number of MYBs failed to exhibit a clear relationship (Table 1). It has been assumed that multiple genome duplication events introduced universal problems in genomics for the distinction and understanding of ohnologs (missing from orthologs) [22,23]. However, according to the earlier reports, there were three whole-genome duplications (WGDs) in the Musa lineage $[18,19]$. Therefore, it is likely that variation in the number of MYBs could be related to WGDs. The insertion of repetitive sequences might lead to the pseudogenization of paralogs. Moreover, the changes in the regulatory and genic sequences could potentially facilitate the inactivation of genes 
or sequence divergence [22,24]. A classification of MYB genes (based on the presence of one, two, three, and four MYB repeats) indicated that there were 73 MYB-1R or MYB-related, 222 MYB-R2R3, 07 MYB-3R, and 03 MYB-4R MYBs of the respective categories in M. acuminata. Similarly, the genome of M. balbisiana possessed 59 MYB-1R or MYB-related, 184 MYB-R2R3, five MYB-3R, and three MYB-4R proteins. A comparison of such categories in close relatives of Musa spp. (i.e., O. sativa [25] and B. distachyon [26] (Figure S1)) showed that there was a comparable number of genes for $1 \mathrm{R}, 3 \mathrm{R}$, and $4 \mathrm{R}$ categories. However, in Muss spp. the number of genes in the R2R3 category was more than two times this amount. A phylogenetic analysis of R2R3-MYBs of 50 eukaryotic organisms [27] suggested that after divergence from a common ancestor the clades could expand distinctively or in a lineage-specific manner.

Table 1. MYB transcription factors identified in plant species of diverse genome sizes.

\begin{tabular}{|c|c|c|c|c|}
\hline Sr\# & Name of Plant & Genome Size (Mbs)* & Number of MYBs & Reference \\
\hline 1 & Arabidopsis thaliana & 119 & 197 & [25] \\
\hline 2 & Sesamum indicum & 270 & 278 & {$[28]$} \\
\hline 3 & Brachypodium distachyon & 270 & 122 & [26] \\
\hline 4 & Citrus sinensis & 301 & 177 & {$[22]$} \\
\hline 5 & Oryza sativa & 373 & 155 & {$[25]$} \\
\hline 6 & Musa acuminata & 332 & 305 & Current study \\
\hline 7 & Musa balbisiana & 430 & 251 & Current study \\
\hline 8 & Physcomitrella patens & 467 & 116 & [29] \\
\hline 9 & Solanum tuberosum & 663 & 158 & [30] \\
\hline 10 & Solanum lycopersicum & 827 & 127 & [31] \\
\hline
\end{tabular}

The lengths of predicted proteins varied from 142 to 1125 and 113 to 996 amino acids in M. acuminata and M. balbisiana, respectively. The relative molecular weight (Mw) ranged between 11.197 and $62.737 \mathrm{kDa}$ in M. acuminata and 10.187 and $63.417 \mathrm{in} \mathrm{M.} \mathrm{balbisiana.} \mathrm{The} \mathrm{predicted} \mathrm{theoretical}$ isoelectric point (PI) was 4.71-10.47 in M. acuminata and 4.48-11.26 in M. balbisiana (Table S2). The MYB proteins of M. acuminata and M. balbisiana were classified into 12 groups (including an intron-less group) depending on the coding sequence for the structure of R2 and R3 MYB repeats (Figure 1). The MYB proteins from O. sativa and A. thaliana were also categorized in these 12 models (MI-MXII). According to Model I, the exon-1 of a MYB gene codes for the first two helices of the R2 MYB repeat, the second exon codes for a part of the third helix in R2 domain, and the first helix in R3 repeats. Similarly, exon-3 is responsible for coding the second and third helix of R3. This is represented in Model Ia. Model I even codes for the three helices of R2 and first helix of R3 (Ib), or the first two helices are coded by intron-1 and the remaining helices are coded by intron-2 (Model Ic). Similarly, there are other variations in gene models, as described by Models II to XI. The R2 and R3 repeats of MaMYB56-like genes (MYB genes with the most number of introns) were classified in Model IX. All the intron-less genes were grouped into MXII. These findings are in accordance with previous investigations [32] that have explained the evolution of MYB genes in lower and higher plant species from a common pool of multiple MYB genes. The group MXII prospectively constitutes the most ancient MYB types [32]. 


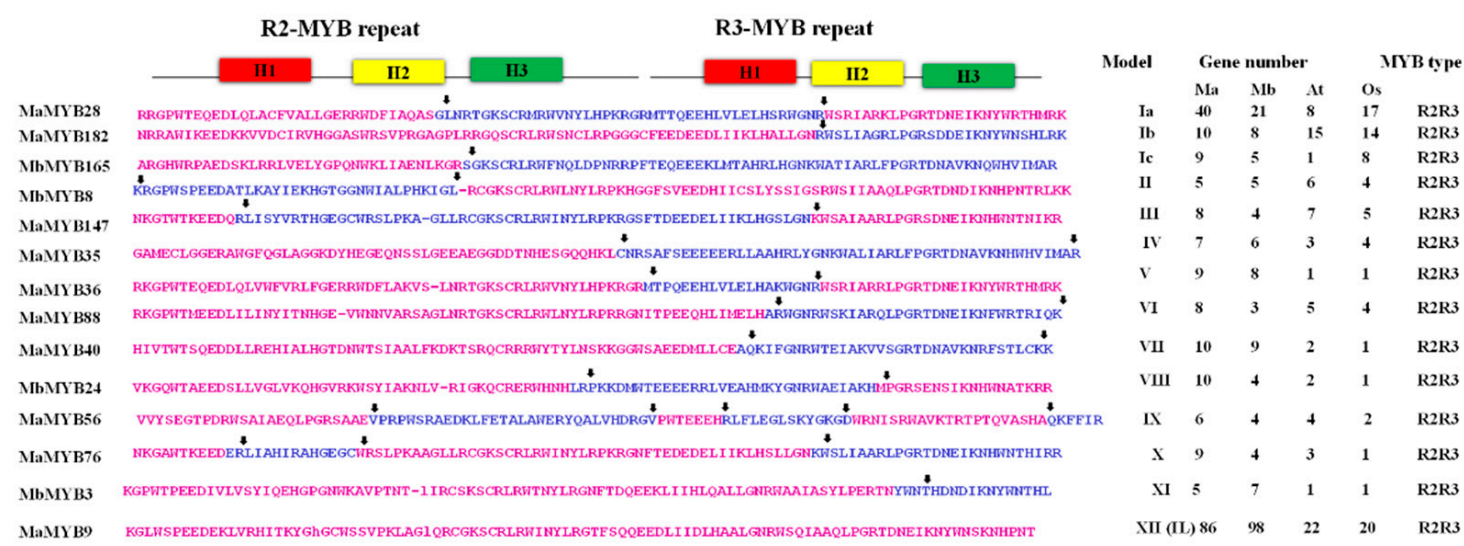

Figure 1. The R2 and R3 repeat coding exon-sequence-based group representation of MYB proteins. The positions of introns are demonstrated with corresponding amino acid residues as arrows, which also represent the position of splicing within the condign sequence. The conserved helices are drawn over the sequences. The numbers of genes in four different plant species are listed on the right side. Intron-less (IL), $\mathrm{Ma}=$ Musa acuminata, $\mathrm{Mb}=$ Musa balbisiana, $\mathrm{Os}=$ Oryza sativa, $\mathrm{At}=$ Arabidopsis thaliana.

The basic structure of the MYB domain consists of almost 50 amino acids with three regularly spaced Trp (W) residues and three $\alpha$-helices: H1, H2, and H3 [33]. Consistent with earlier reports, $M a M Y B$ and $M b M Y B$ genes harbored the typical MYB domain and contained a characteristic amino acid with a series of highly conserved Trp residues. These residues are considered a landmark of MYB proteins and play a significant role in the sequence-specific binding of DNA (Figure 2). The second Trp residue in the R3MYYB domain of M. balbisiana was replaced by other amino acids, which may have affected the binding affinity of the DNA. In addition to highly conserved Trp residues, other highly conserved residues were observed in more than $90 \%$ of MYB domains from both M. acuminat $a$ and M. balbisiana.

Introns usually undergo rapid changes and are often neutral to selection during the process of evolution; hence, higher sequence-similarity between orthologous introns show a functional restraint in the evolutionary process [34]. The intron-exon structure of R2R3-MYB repeats in higher plants is conserved, and intron-containing genes have previously been sub-grouped into four to six groups $[17,25,35]$. In this study, exon-intron organization was examined to better understand the structural organization, which was found to be similar in both banana species. Moreover, it was observed that most of R2R3-MYB genes had 1 to 10 introns in the coding sequence. Similarly, seven of the 1R-MYBs had three introns while six of the R3-MYB genes had four introns (Figure 2a,b). These outcomes exhibit the occurrence of well-preserved configurations within the MaMYB and MbMYB subfamilies and elevated sequence variation amongst diverse groups of the two banana species. 

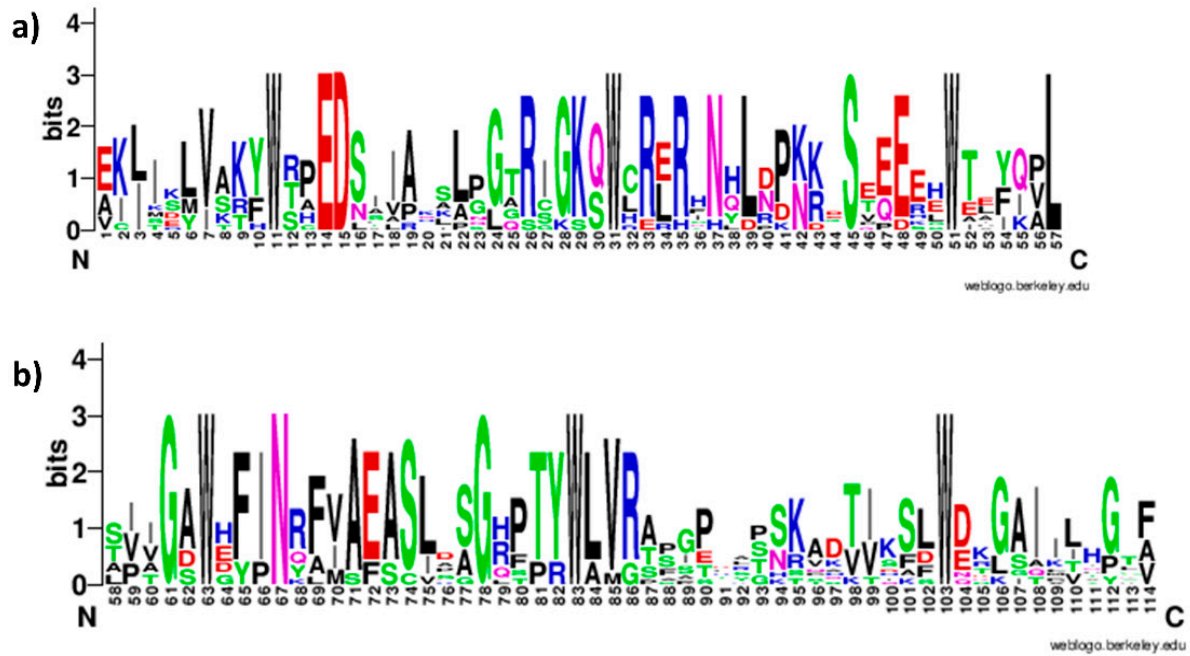

(a) Musa acuminata

a)

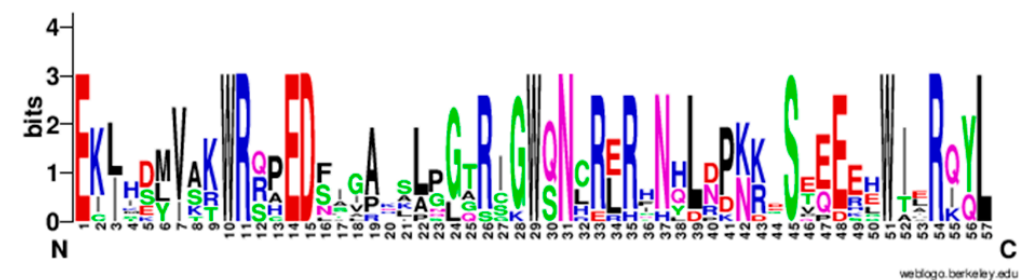

b)

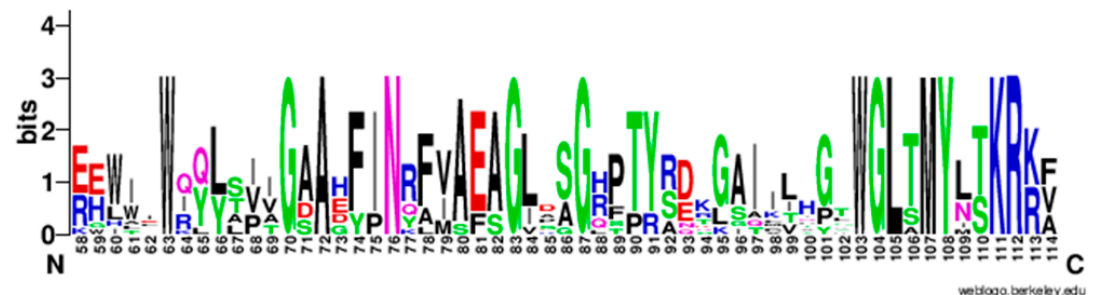

(b) Musa balbisiana

Figure 2. Sequence logos of the conserved R2 and R3 repeats of the MYB domain across all R2R3-MYB proteins in the (a) M. acuminata and (b) M. balbisiana genomes. The sequence logos are based on global alignments of R2/R3 MYB repeats from M. acuminata and M. balbisiana R2R3-MYB domains.

\subsection{Chromosomal Distribution and Duplication}

The genomic sequences of MYB genes were taken from NCBI, and the chromosomal location of MYB genes on each chromosome was mapped by MapChart. Analysis of genomic location displayed that the banana MYB genes were randomly distributed throughout 11 linkage groups (Figure 3a,b). In M. acuminata, out of 305 genes, 283 genes were mapped on 11 chromosomes. Most MaMYB genes were found on chromosome 6 ( 35 genes), while chromosome 3 had the least number of genes (18). Chromosomal positions of 17 genes (MaMYB12, MaMYB13, MaMYB14, MaMYB278, MaMYB279, MaMYB280, MaMYB281, МaMYB282, МaMYB283, MaMYB284, MaMYB285, MaMYB286, MaMYB287, MaMYB288, MaMYB289, MaMYB290, MaMYB291) were unavailable. They were present on the scaffolds, which have not yet assigned to any linkage group (Figure 3a). 


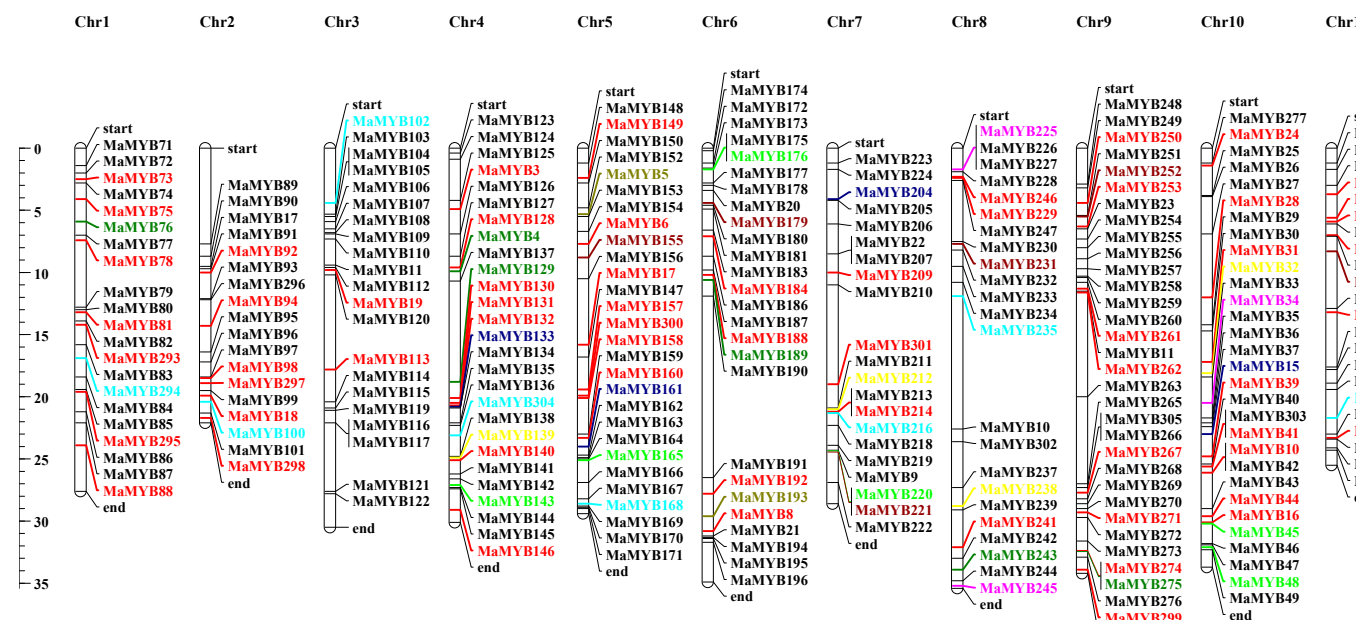

(a)
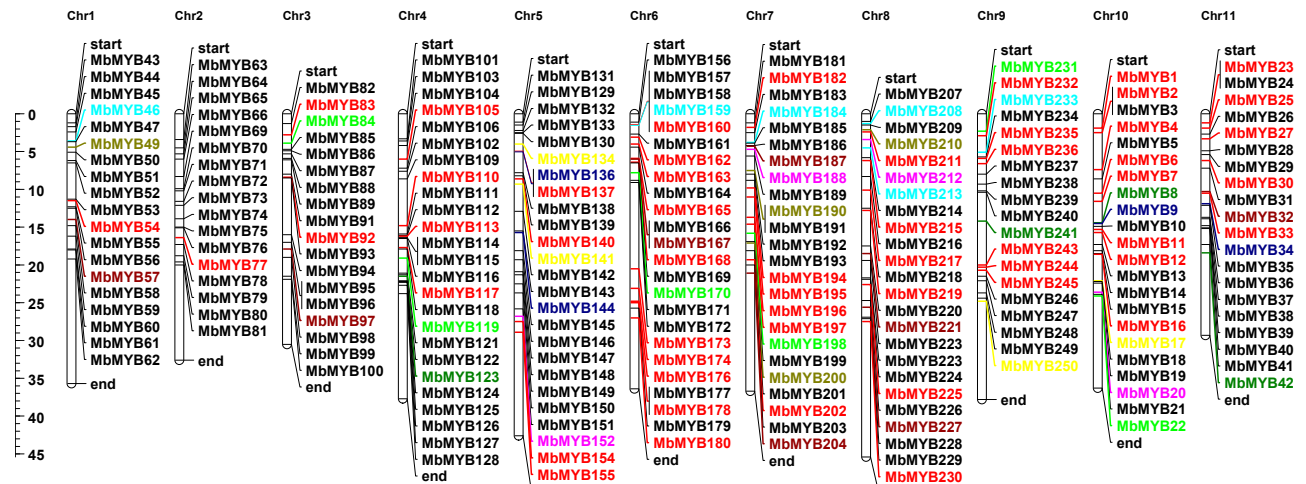

(b)

Figure 3. The arrangement of MYB genes on the chromosomes of (a) Musa acuminata and (b) Musa balbisiana. The chromosome numeral (Chr) is shown above every chromosome. The arrangement is designated on a centimorgan (cM) scale. The red color represents segmental duplication. Similar colors (other than red) represent the tandem duplicated genes.

Out of 251 genes in M. balbisiana, 237 MYB genes were mapped on 11 chromosomes (Figure 3b). Like M. acuminata, all MYB genes of M. balbisiana were randomly distributed. Most MYB genes were present on chromosome 4 and 5 ( 25 genes on each). Chromosome 2 carried the least number of MYB genes (17 genes). It is interesting to note that chromosome 2 in both banana species carried the least number of MYB genes (Figure 3a,b). There were 14 MbMYB genes (MbMYB48, MbMYB67, MbMYB68, MbMYB90, MbMYB107, MbMYB108, MbMYB120, MbMYB135, MbMYB153, MbMYB175, MbMYB205, MbMYB206, MbMYB242, MbMYB251) present in scaffold regions.

Almost $26 \%$ of MaMYB genes were duplicated representing either segmental duplication (51 genes) or tandem duplication (30 genes) (Table S4). Similarly, almost 24\% of MbMYB genes experienced duplication segmentally (38) or tandemly (23). The non-synonymous (Ka)/synonymous (Ks) ratio was calculated for all the tandemly and segmentally duplicated $M Y B$ gene-pairs as less than 1 or greater than 1 , respectively. This revealed that the gene pairs with a $\mathrm{Ka} / \mathrm{Ks}$ ratio of less than 1 were subjected to purifying selection, and those with a ratio of more than 1 experienced positive selection $[36,37]$. In the evolutionary process, most plants experienced one or more ancient polyploidies. Gene duplication has long been known to occur during plant evolution, thereby contributing to the formation of new gene functions, expansion of large gene families, and origins of evolutionary novelty [38]. The genome Arabidopsis has undergone two current whole-genome duplications (WGD; $\alpha$ and $\beta$ ) in the lineage of Brassicaceae [39]. A previous report classified the chromosomal duplication of Arabidopsis into 
three types based on the duplication times of $\alpha, \beta$, and $\gamma$ [39]. Accordingly, whole-genome analysis of the MYB gene family in Glycine max, Populus trichocarpa, Oryza sativa, and Zea mays showed that multiple tandem and segmental duplications events play a crucial role in the expansion of the MYB gene family [17]. High segmental and low tandem duplications have commonly been present in the MYB gene family in plants [38], which is supported by several publications $[16,38,40]$. Copies of genes evolved by segmental duplications are more often reserved in the slowly evolving MYB gene family than in tandem duplication [38]. Higher proportions of segmental duplication has revealed that the expansion of MYB genes in both M. acuminata and M. balbisiana is due to the segmental duplication [41]. Moreover, a lower tandem gene duplication is potentially associated with gene families involved in housekeeping or key regulatory functions [38,42].

\subsection{Phylogenetic Analysis of Musa acuminata and Musa balbisiana}

To examine the phylogenetic relationship of banana MYB genes, a phylogenetic tree was constructed from protein sequences of A. thaliana, O. sativa M. acuminata, and M. balbisiana (Figure 4). The topologies of the phylogenetic tree were similar to previously reported results from a phylogenetic analysis comparing O. sativa and A. thaliana $[25,26,30]$.

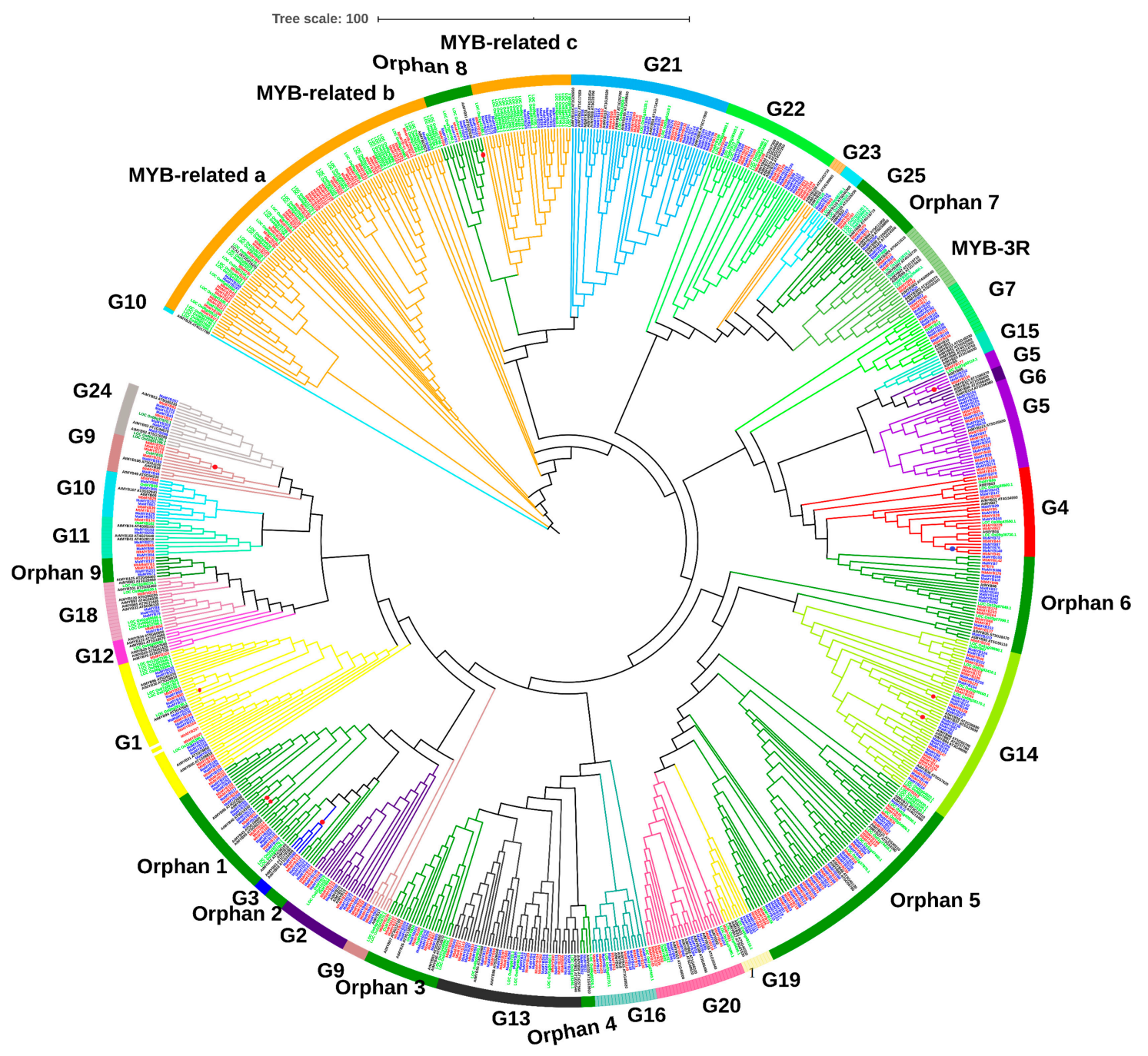

Figure 4. A phylogenetic tree of MYB proteins based on functional studies. MYB genes of $M$. acuminata (Ma, blue), M. balbisiana (Mb, red), O. sativa (Os, green), and A. thaliana (At, black) were used. The amino acid sequence was aligned by CLUSTALW and the phylogenetic tree was made using the neighbor-joining method. 
Comparative analysis of MYB genes in these genomes indicated that genes with orthologous relationships happened to be grouped in the phylogenetic tree rather than in paralogs. This reveals that a higher diversification of MYB genes occurred in the ancestor species during the evolutionary process [27,43]. According to our results and based on previously reported analyses of $O$. sativa and A. thaliana MYB proteins [25,44], the MYB proteins of $M$. acuminata and M. balbisiana could be classified into 25 MYB-R2R3 subgroups (i.e., G1 to G25), nine orphan or atypical subgroups, three MYB-related subgroups $(a, b, c)$, and one MYB-3R subgroup. The phylogenetic analysis showed that there was an unequal representation of members from M. acuminata, M. balbisiana, O. sativa, and $A$. thaliana. For example, G6, G12, and G23 did not contain any member from M. acuminata or M. balbisiana. This might indicate a loss of corresponding members after divergence [45]. Otherwise, it is possible that they were lost in the assembly or annotation of the banana genome. Moreover, some groups (like atypical 2) included members only from M. acuminata and M. balbisiana [46]. It has been suggested that such genes might have a specialized role in banana, and that they were either lost in O. sativa and A. thaliana, or acquired in these fruit species, after divergence from common ancestors [27]. Such groups are good candidates for phylogenetic and functional studies of banana. Similarly, MYBs from both species of banana shared most of the groups except G15, G19, atypical 4, atypical 5, and MYB-related c groups. Members of such groups are important candidates for species-specific functional investigations (Table 2).

Table 2. Details of the number of genes in each group of phylogenetic trees for each species understudy.

\begin{tabular}{|c|c|c|c|c|}
\hline Short Name & МаMYв & MbMYB & AtMYB & OsMYB \\
\hline G1 & 16 & 10 & 5 & 9 \\
\hline G2 & 7 & 8 & 3 & 3 \\
\hline G3 & 1 & 1 & 2 & 0 \\
\hline G4 & 10 & 8 & 4 & 4 \\
\hline G5 & 17 & 11 & 2 & 0 \\
\hline G6 & 0 & 0 & 4 & 0 \\
\hline G7 & 8 & 4 & 3 & 1 \\
\hline G9 & 3 & 4 & 3 & 1 \\
\hline G10 & 5 & 3 & 2 & 1 \\
\hline G11 & 5 & 2 & 3 & 1 \\
\hline G12 & 0 & 0 & 7 & 0 \\
\hline G13 & 27 & 14 & 9 & 11 \\
\hline G14 & 23 & 15 & 6 & 7 \\
\hline G15 & 0 & 1 & 5 & 0 \\
\hline G16 & 7 & 6 & 3 & 2 \\
\hline G18 & 4 & 2 & 5 & 5 \\
\hline G19 & 4 & 0 & 3 & 2 \\
\hline G20 & 9 & 8 & 6 & 3 \\
\hline G21 & 23 & 11 & 9 & 3 \\
\hline G22 & 12 & 11 & 4 & 6 \\
\hline G23 & 0 & 0 & 3 & 1 \\
\hline
\end{tabular}


Table 2. Cont.

\begin{tabular}{ccccc}
\hline Short Name & MaMYB & MbMYB & AtMYB & OsMYB \\
\hline G24 & 7 & 2 & 3 & 3 \\
\hline G25 & 3 & 4 & 5 & 3 \\
\hline Atypical & 11 & 7 & 3 & 6 \\
\hline Atypical 2 & 4 & 2 & 0 & 0 \\
\hline Atypical 3 & 7 & 6 & 4 & 5 \\
\hline Atypical 4 & 2 & 0 & 1 & 1 \\
\hline Atypical 5 & 0 & 2 & 6 & 2 \\
\hline Atypical 6 & 6 & 22 & 3 & 2 \\
\hline Atypical 7 & 13 & 11 & 7 & 4 \\
\hline Atypical 8 & 5 & 4 & 1 & 24 \\
\hline MYB related a & 2 & 4 & 1 & 17 \\
\hline MYB related b & 4 & 3 & 0 & 17 \\
\hline MYB related c & 8 & 0 & 0 &
\end{tabular}

The phylogenetic results were consistent with recent reports $[26,32,47]$ showing that various groups included more MYBs from Arabidopsis than M. acuminata, M. balbisiana, and O. sativa. These results are in accordance with the current information that Arabidopsis underwent more duplication events after divergence of the last common ancestor from these three close species. This suggests that the existence of species-specific MYBs were either acquired in the Musa lineages after divergence from common ancestors, or lost in Arabidopsis [25]. The physiological and anatomical differences between M. acuminata, M. balbisiana, and Arabidopsis strengthen the prediction that some members of the MYB family may have been differentially expanded. Outside and inside of these functional clades, M. acuminata and M. balbisiana MYB genes appeared as clusters or gene pairs (Figure 4). Moreover, comparative phylogenetic analysis between M. acuminata and M. balbisiana revealed a high level of conservation between the two genomes of banana.

\subsection{Expression Profile of MYB Genes during Fruit Ripening}

MYB genes have been widely studied and found to be involved in numerous plant-specific processes including development, metabolism, and gene expression regulation for biotic and abiotic stress response [17,33-35]. Therefore, to gain more insight into the role of MYB genes in the ripening of banana (Figure 5), relative real-time RT-qPCR was performed to evaluate the transcript abundance of target genes in fruit ripening.

After the application of acetylene, both banana species responded differentially. Banana finger-drop was observed in M. acuminata after three days. However, it was delayed in M. balbisiana, which is considered less sensitive to acetylene application [48]. The RNA-seq data were accessed from the Banana Genome Hub to select MYB genes responsive to acetylene application [49]. For validation, mostly those genes were selected that shared a clade in the phylogenetic tree. These MYB genes (from both species) are strong homologs, as most of them are present on same monophyletic taxon (represented by colored dots in phylogenetic tree). Twelve MYB genes were selected (from each species) for validation through qPCR (Table S3). The results of qPCR were mostly associated with RNA-seq expression data. However, some differences were also found. Although we applied similar growth conditions, such differences could be related to genotypic differences, plant age, or even sampling time in a day. The real-time expression profile showed diverse expression patterns. The transcript abundance of some MYB genes (MaMYB 17, 98, 101, 116, 151, 207, and 214) increased with time 
(Figure 6a). Similarly, the expression of MbMYB26, 49, 80, 128, 135, and 191 was increased, while the expression of $M b M Y B 76$ and 151 was decreased with time (Figure 6b). This suggests a potential involvement of these genes in fruit ripening or development. In the phylogenetic tree, these genes were clustered in G1 (MaMYB101/MbMYB80), G3 (MaMYB98/MbMYB76), G4 (MaMYB76/MbMYB49), G5 (MaMYB17/MbMYB135), G9 (MaMYB214/MbMYB151), G14 (MaMYB6/MbMYB128), as well as in the Atypical/Orphan 1 (MaMYB151/MbMYB57) and Atypical/Orphan 8 (MaMYB207/MbMYB191) groups (Figure 4). Members of these groups are involved in several processes related to plant development [44]. In G4, MYB proteins from banana (MaMYB76 and MbMYB49) clustered with AtMYB04 (Figure 4), which is involved in flavonoid biosynthesis [50,51]. Similarly, AtMYB5 (shared clade with MaMYB17 and MbMYB135 in G5) is involved in regulating PA biosynthesis [52-54]. The expression of MaMYB6 decreased from day 0 to 7 (Figure 6a). The $M Y B 6$ is a transcriptional repressor that negatively regulates the ripening of banana fruit by obstructing starch degradation [55]. A progressive downregulation of MYB6 transcripts correlates with a conserved repressive role of this gene.
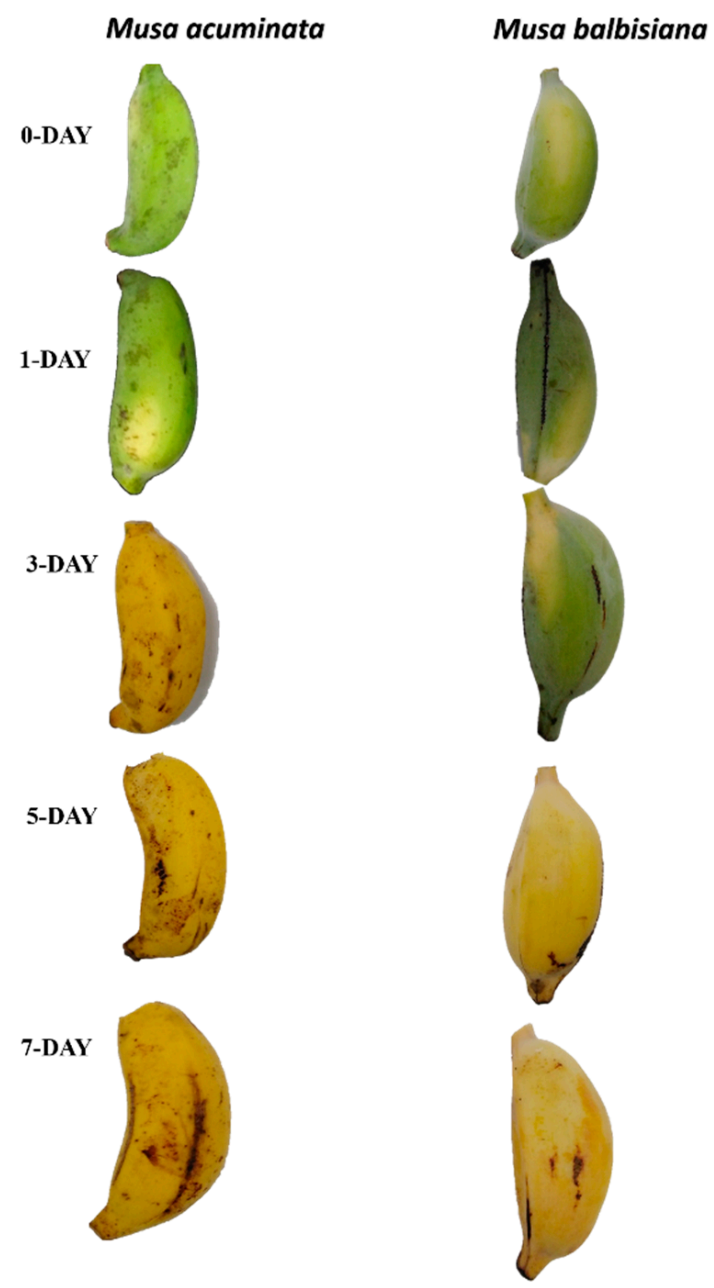

Figure 5. Comparison of M. acuminata and M. balbisiana fruits at different stages of fruit ripening. 

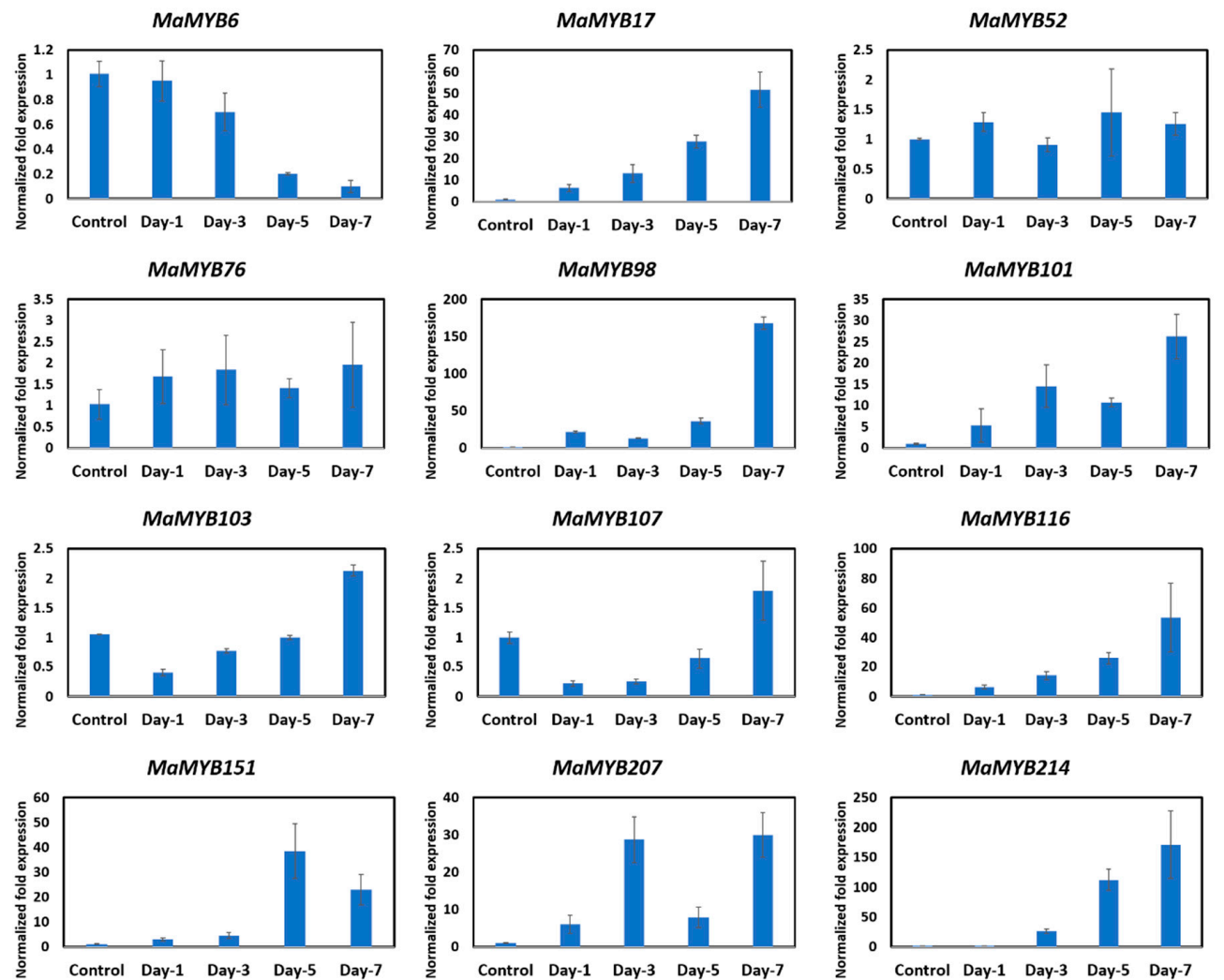

(a)
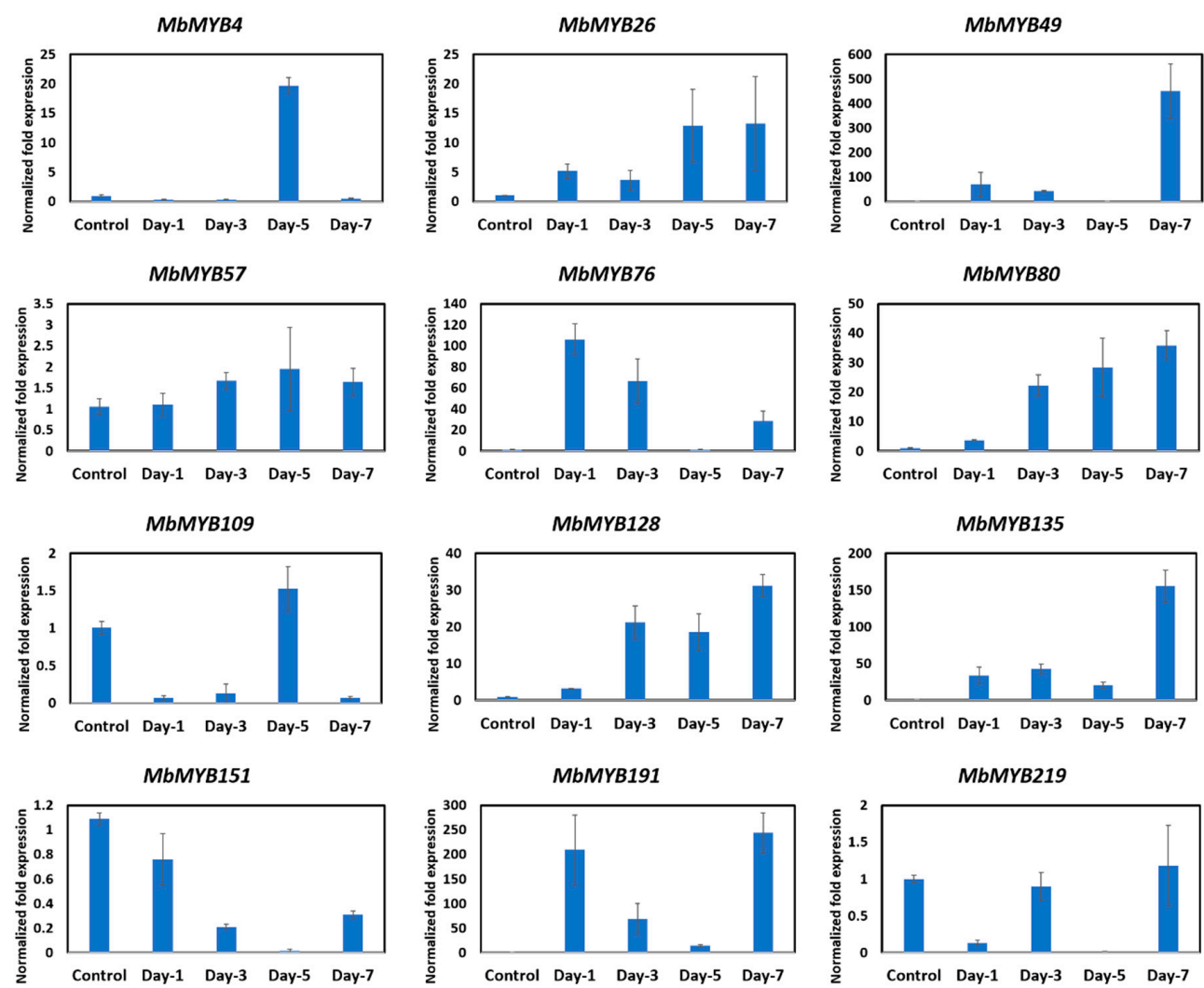

(b)

Figure 6. Relative RT-qPCR assay of MYB genes in (a) M. acuminata and (b) M. balbisiana during ripening before and after the application of acetylene. The default expression value for each gene was from non-treated plants. Error bars indicate the standard deviation from the mean (three replicates). 


\section{Material and Methods}

\subsection{Sequence Database Searches}

The Banana Genome Hub (https://banana-genome-hub.southgreen.fr/) was used to retrieve the sequences of both banana species (i.e., M. acuminata and M. balbisiana). The protein sequences for MYBs from A. thaliana and O. sativa were obtained from the Arabidopsis Information Resource (TAIR release 10, http://www.arabidopsis.org), Rice Genome Annotation Project database (RGAP release 7, http://rice.plantbiology.msu.edu/index.shtml), and plant transcription factor database (PlantTFDB: http://planttfdb.cbi.pku.edu.cn/). The IDs and names of genes were listed in Table S2.

\subsection{The Identification and Chromosomal Mapping of MYB TFs}

The Pfam database (http://pfam.xfam.org/) was accessed to retrieve the SANT domain (PF00249) representing the MYB binding domain. It was used to screen MYB genes in both species of banana using HMMER (v3.1b2) (http://hmmer.org). An e-value of less than 1e-10 was used as a cut off threshold. For further confirmation of the identity of each sequence as MYB, these sequences were also subjected to a screening in SMART (http://smart.embl-heidelberg.de/) and CDD (http://www.ncbi.nlm.nih.gov/ Structure/cdd/wrpsb.cgi). The gene structure display server (GSDS) (http://gsds.cbi.pku.edu.cn/) was used to obtain a schematic representation of all gene structures. The molecular weight ad isoelectric point was investigated through Expasy (http://web.expasy.org/protparam/). The MapChart program (http://www.biometris.wur.nl/UK/Software/MapChart/download) was used to demonstrate the physical location of the MYB genes on chromosomes of M. acuminata and M. balbisiana. The gene duplication events were determined through the DNAsp program using the rates of non-synonymous (Ka) and synonymous (Ks) substitutions.

\subsection{Phylogenetic Analysis}

For this analysis, multiple sequence alignment was performed through ClustalW in Mega 7.0 software. Later on, a phylogenetic tree was built using the neighbor-joining likelihood method with 1000 bootstrap replicates. MYB protein sequences from M. balbisiana, M. acuminate, A. thaliana, and $O$. sativa were used for the analysis of phylogenetic and evolutionary relationships.

\subsection{Plant Material and Stress Imposition}

Fruits were harvested from M. acuminata and M. balbisiana at the green maturation stage. The plants were grown in an orchard in Danzhou, Hainan, China. A 500× diluted solution of 30\% acetylene (dissolved in ethanol) was applied to the detached fruits. Acetylene is an analog of ethylene, which is used for artificially ripening fruits [56]. It has a lesser biological activity than ethylene and there is no significant difference in sensory attributes between bananas treated with ethylene and acetylene [57]. Acetylene was applied for one, three, five, and seven days after washing the fruits with clean water. The control samples were collected before treatment. The treated and non-treated fruits were kept at $22{ }^{\circ} \mathrm{C}$ under dark conditions.

\subsection{RNA Extraction and Quantitative Real-Time PCR (qRT-PCR)}

An RNA extraction Kit (RNAprep Pur Plant Kit for polysaccharides and polyphenolic-rich samples from TIANGEN Biotech, Beijing) was used for total RNA extraction from banana fruits according to the instruction of the manufacturer. RNA was quantified using Thermo Nanodrop 2000 (Thermo Fisher Scientific, Massachusetts). One microgram of RNA was reverse-transcribed for cDNA synthesis using an All-in-One First-Strand Synthesis kit (Monad, Jiangsu, China cDNA). The cDNA was stored at $-20{ }^{\circ} \mathrm{C}$ for further use. The qRT-PCR analysis was performed using an Applied Biosystems StepOnePlus Real-Time PCR System and TB Green ${ }^{\mathrm{T}}$ Premix Ex Taq $^{\mathrm{T}}$ II, Tli RNaseH plus kit (Takara). The Oligo Calculator tool (http://mcb.berkeley.edu/labs/krantz/tools/oligocalc.html) 
was used to design gene-specific primers, and specificity of primers was verified by NCBI-primer BLAST program (https://www.ncbi.nlm.nih.gov/tools/primer-blast/) (Table S3). Expression analysis of MYB genes was repeated three times and the ribosomal protein S (RSP2) was used as the house-keeping gene [58].

\section{Conclusions}

Banana is globally consumed and exhibits a vital role in food security for millions of people around the world. The MYB genes of M. acuminata and M. balbisiana were classified into 12 groups (MI-MXII) based on intron and exon structure models of the MYB domain sequences. MaMYB and MbMYB genes were randomly distributed on 11 chromosomes. Phylogenetic analysis of the MYB family among banana and plant species models indicated the functional divergence during evolution. The current study reports the existence of species-specific MYBs that are potential candidates for functional characterization. Of the two species, M. acuminata seemed more sensitive to acetylene than M. balbisiana did. The transcript abundance of MaMYBs (i.e., MaMYB17, 98, 101, 116, 151, 207, and 214) and MbMYBs (MbMYB26, 49, 80, 128, 135, and 191) was increased in response to acetylene or development. The findings of this study will facilitate future studies aimed at the functional characterization of MYB TFs in banana.

Supplementary Materials: The following are available online at http://www.mdpi.com/2223-7747/9/4/413/s1, Figure S1: Phylogenetic tree of plant species., Table S1: Details MYB genes studied in different plant species. Table S2: Gene Ids and their sequence features of MYB genes. Table S3: List of primers used in this study. Table S4: Segmental and tandem duplication in MYB genes in Musa acuminata and Musa balbisiana.

Author Contributions: F.A. and L.T. designed the experiment; L.T., U.I., H.S., N.N.W.H. and Z.C. analyzed the data; L.T, U.I and F.A. wrote the manuscript; Y.G. and F.A. revised the manuscript. All authors read and approved the final manuscript.

Funding: This research was funded by Central Public-interest Scientific Institution Basal Research Fund for Chinese Academy of Tropical Agricultural Sciences, grant number 1630032014048 and the APC was funded by Lin Tan.

Conflicts of Interest: The authors declare no conflict of interest.

\section{References}

1. Riechmann, J.L. Arabidopsis transcription factors: Genome-wide comparative analysis among eukaryotes. Science 2000, 290, 2105-2110. [CrossRef] [PubMed]

2. Dubos, C.; Stracke, R.; Grotewold, E.; Weisshaar, B.; Martin, C.; Lepiniec, L. MYB transcription factors in Arabidopsis. Trends Plant Sci. 2010, 15, 573-581. [CrossRef] [PubMed]

3. Jin, H.; Martin, C. Multifunctionality and diversity within the plant MYB-gene family. Plant Mol. Biol. 1999, 41, 577-585. [CrossRef]

4. Kanei-Ishii, C.; Sarai, A.; Sawazaki, T.; Nakagoshi, H.; He, D.N.; Ogata, K.; Nishimura, Y.; Ishii, S. The tryptophan cluster: A hypothetical structure of the DNA-binding domain of the myb protooncogene product. J. Biol. Chem. 1990, 265, 19990-19995. [PubMed]

5. Ogata, K.; Hojo, H.; Aimoto, S.; Nakai, T.; Nakamura, H.; Sarai, A.; Ishii, S.; Nishimura, Y. Solution structure of a DNA-binding unit of Myb: A helix-turn-helix-related motif with conserved tryptophans forming a hydrophobic core. Proc. Natl. Acad. Sci. USA 1992, 89, 6428-6432. [CrossRef] [PubMed]

6. Martin, C.; Paz-Ares, J. MYB transcription factors in plants. Trends Genet. 1997, 13, 67-73. [CrossRef]

7. Stracke, R.; Werber, M.; Weisshaar, B. The R2R3-MYB gene family in Arabidopsis thaliana. Curr. Opin. Plant Biol. 2001, 4, 447-456. [CrossRef]

8. Paz-Ares, J.; Ghosal, D.; Wienand, U.; Peterson, P.A.; Saedler, H. The regulatory c1 locus of Zea mays encodes a protein with homology to myb proto-oncogene products and with structural similarities to transcriptional activators. EMBO J. 1987, 6, 3553-3558. [CrossRef]

9. Li, C.; Ng, C.K.-Y.; Fan, L.-M. MYB transcription factors, active players in abiotic stress signaling. Environ. Exp. Bot. 2015, 114, 80-91. [CrossRef] 
10. Ma, D.; Constabel, C.P. MYB repressors as regulators of phenylpropanoid metabolism in plants. Trends Plant Sci. 2019, 24, 275-289. [CrossRef]

11. Hatlestad, G.J.; Akhavan, N.A.; Sunnadeniya, R.M.; Elam, L.; Cargile, S.; Hembd, A.; Gonzalez, A.; McGrath, J.M.; Lloyd, A.M. The beet $Y$ locus encodes an anthocyanin MYB-like protein that activates the betalain red pigment pathway. Nat. Genet. 2015, 47, 92-96. [CrossRef]

12. Yao, G.; Ming, M.; Allan, A.C.; Gu, C.; Li, L.; Wu, X.; Wang, R.; Chang, Y.; Qi, K.; Zhang, S.; et al. Map-based cloning of the pear gene MYB114 identifies an interaction with other transcription factors to coordinately regulate fruit anthocyanin biosynthesis. Plant J. 2017, 92, 437-451. [CrossRef] [PubMed]

13. Medina-Puche, L.; Molina-Hidalgo, F.J.; Boersma, M.; Schuurink, R.C.; López-Vidriero, I.; Solano, R.; Franco-Zorrilla, J.-M.; Caballero, J.L.; Blanco-Portales, R.; Muñoz-Blanco, J. An R2R3-MYB transcription factor regulates eugenol production in ripe strawberry fruit receptacles. Plant Physiol. 2015, 168, 598-614. [CrossRef]

14. Lau, S.-E.; Schwarzacher, T.; Othman, R.Y.; Harikrishna, J.A. dsRNA silencing of an R2R3-MYB transcription factor affects flower cell shape in a Dendrobium hybrid. BMC Plant Biol. 2015, 15, 194. [CrossRef] [PubMed]

15. Cheng, H.-Q.; Han, L.-B.; Yang, C.-L.; Wu, X.-M.; Zhong, N.-Q.; Wu, J.-H.; Wang, F.-X.; Wang, H.-Y.; Xia, G.-X. The cotton MYB108 forms a positive feedback regulation loop with CML11 and participates in the defense response against Verticillium dahliae infection. J. Exp. Bot. 2016, 67, 1935-1950. [CrossRef] [PubMed]

16. Sheehan, H.; Moser, M.; Klahre, U.; Esfeld, K.; Dell'Olivo, A.; Mandel, T.; Metzger, S.; Vandenbussche, M.; Freitas, L.; Kuhlemeier, C. MYB-FL controls gain and loss of floral UV absorbance, a key trait affecting pollinator preference and reproductive isolation. Nat. Genet. 2016, 48, 159-166. [CrossRef] [PubMed]

17. Matus, J.T.; Aquea, F.; Arce-Johnson, P. Analysis of the grape MYB R2R3 subfamily reveals expanded wine quality-related clades and conserved gene structure organization across Vitis and Arabidopsis genomes. BMC Plant Biol. 2008, 8, 83. [CrossRef]

18. D’Hont, A.; Denoeud, F.; Aury, J.-M.; Baurens, F.-C.; Carreel, F.; Garsmeur, O.; Noel, B.; Bocs, S.; Droc, G.; Rouard, M.; et al. The banana (Musa acuminata) genome and the evolution of monocotyledonous plants. Nature 2012, 488, 213-217. [CrossRef]

19. Wang, Z.; Miao, H.; Liu, J.; Xu, B.; Yao, X.; Xu, C.; Zhao, S.; Fang, X.; Jia, C.; Wang, J.; et al. Musa balbisiana genome reveals subgenome evolution and functional divergence. Nat. Plants 2019, 5, 810-821. [CrossRef]

20. Davey, M.W.; Gudimella, R.; Harikrishna, J.A.; Sin, L.W.; Khalid, N.; Keulemans, J. A draft Musa balbisiana genome sequence for molecular genetics in polyploid, inter- and intra-specific Musa hybrids. BMC Genom. 2013, 14, 683. [CrossRef]

21. Wang, X.; Niu, Q.-W.; Teng, C.; Li, C.; Mu, J.; Chua, N.-H.; Zuo, J. Overexpression of PGA37/MYB118 and MYB115 promotes vegetative-to-embryonic transition in Arabidopsis. Cell Res. 2009, 19, 224-235. [CrossRef] [PubMed]

22. Hou, X.-J.; Li, S.-B.; Liu, S.-R.; Hu, C.-G.; Zhang, J.-Z. Genome-Wide Classification and evolutionary and expression analyses of citrus MYB transcription factor families in sweet orange. PLoS ONE 2014, 9, e112375. [CrossRef] [PubMed]

23. Catchen, J.M.; Conery, J.S.; Postlethwait, J.H. Automated identification of conserved synteny after whole-genome duplication. Genome Res. 2009, 19, 1497-1505. [CrossRef] [PubMed]

24. Zhang, Z.; Zhou, L.; Wang, P.; Liu, Y.; Chen, X.; Hu, L.; Kong, X. Divergence of exonic splicing elements after gene duplication and the impact on gene structures. Genome Biol. 2009, 10, R120. [CrossRef] [PubMed]

25. Katiyar, A.; Smita, S.; Lenka, S.; Rajwanshi, R.; Chinnusamy, V.; Bansal, K. Genome-wide classification and expression analysis of MYB transcription factor families in rice and Arabidopsis. BMC Genom. 2012, 13, 544. [CrossRef]

26. Chen, S.; Niu, X.; Guan, Y.; Li, H. Genome-wide analysis and expression profiles of the MYB genes in Brachypodium distachyon. Plant Cell Physiol. 2017, 58, 1777-1788. [CrossRef]

27. Du, H.; Liang, Z.; Zhao, S.; Nan, M.-G.; Tran, L.-S.P.; Lu, K.; Huang, Y.-B.; Li, J.-N. The evolutionary history of R2R3-MYB proteins across 50 eukaryotes: New insights into subfamily classification and expansion. Sci. Rep. 2015, 5, 11037. [CrossRef]

28. Mmadi, M.; Dossa, K.; Wang, L.; Zhou, R.; Wang, Y.; Cisse, N.; Sy, M.; Zhang, X. Functional characterization of the versatile myb gene family uncovered their important roles in plant development and responses to drought and waterlogging in sesame. Genes 2017, 8, 362. [CrossRef] 
29. Pu, X.; Yang, L.; Liu, L.; Dong, X.; Chen, S.; Chen, Z.; Liu, G.; Jia, Y.; Yuan, W.; Liu, L. Genome-wide analysis of the MYB transcription factor superfamily in Physcomitrella patens. Int. J. Mol. Sci. 2020, 21, 975. [CrossRef]

30. Sun, W.; Ma, Z.; Chen, H.; Liu, M. MYB gene family in potato (Solanum tuberosum L.): Genome-wide identification of hormone-responsive reveals their potential functions in growth and development. Int. J. Mol. Sci. 2019, 20, 4847. [CrossRef]

31. Li, Z.; Peng, R.; Tian, Y.; Han, H.; Xu, J.; Yao, Q. Genome-wide identification and analysis of the myb transcription factor superfamily in Solanum lycopersicum. Plant Cell Physiol. 2016, 57, 1657-1677. [CrossRef] [PubMed]

32. Zhou, C.; Chen, Y.; Wu, Z.; Lu, W.; Han, J.; Wu, P.; Chen, Y.; Li, M.; Jiang, H.; Wu, G. Genome-wide analysis of the MYB gene family in physic nut (Jatropha curcas L.). Gene 2015, 572, 63-71. [CrossRef] [PubMed]

33. Cao, Y.; Han, Y.; Li, D.; Lin, Y.; Cai, Y. MYB transcription factors in chinese pear (Pyrus bretschneideri Rehd.): Genome-wide identification, classification, and expression profiling during fruit development. Front. Plant Sci. 2016, 7, 577. [CrossRef] [PubMed]

34. Roy, S. Function of MYB domain transcription factors in abiotic stress and epigenetic control of stress response in plant genome. Plant Signal. Behav. 2016, 11, e1117723. [CrossRef]

35. Jiang, H.; Wu, P.; Zhang, S.; Song, C.; Chen, Y.; Li, M.; Jia, Y.; Fang, X.; Chen, F.; Wu, G. Global analysis of gene expression profiles in developing physic nut (Jatropha curcas L.) seeds. PLoS ONE 2012, 7, e36522. [CrossRef]

36. Roth, C.; Liberles, D.A. A systematic search for positive selection in higher plants (Embryophytes). BMC Plant Biol. 2006, 6, 12. [CrossRef]

37. Hurst, L.D. The Ka/Ks ratio: Diagnosing the form of sequence evolution. Trends Genet. 2002, 18, $486-487$. [CrossRef]

38. Cannon, S.B.; Mitra, A.; Baumgarten, A.; Young, N.D.; May, G. The roles of segmental and tandem gene duplication in the evolution of large gene families in Arabidopsis thaliana. BMC Plant Biol. 2004, 4, 10. [CrossRef]

39. del Pozo, J.C.; Ramirez-Parra, E. Whole genome duplications in plants: An overview from Arabidopsis. J. Exp. Bot. 2015, 66, 6991-7003. [CrossRef]

40. Li, Y.; Lin-Wang, K.; Liu, Z.; Allan, A.C.; Qin, S.; Zhang, J.; Liu, Y. Genome-wide analysis and expression profiles of the StR2R3-MYB transcription factor superfamily in potato (Solanum tuberosum L.). Int. J. Biol. Macromol. 2020, 148, 817-832. [CrossRef]

41. Du, H.; Yang, S.-S.; Liang, Z.; Feng, B.-R.; Liu, L.; Huang, Y.-B.; Tang, Y.-X. Genome-wide analysis of the MYB transcription factor superfamily in soybean. BMC Plant Biol. 2012, 12, 106. [CrossRef] [PubMed]

42. Ahmad, B.; Azeem, F.; Ali, M.A.; Nawaz, M.A.; Nadeem, H.; Abbas, A.; Batool, R.; Atif, R.M.; Ijaz, U.; Nieves-Cordones, M.; et al. Genome-wide identification and expression analysis of two component system genes in Cicer arietinum. Genomics 2019, 112, 1371-1383. [CrossRef] [PubMed]

43. Feng, G.; Burleigh, J.G.; Braun, E.L.; Mei, W.; Barbazuk, W.B. Evolution of the 3R-MYB gene family in plants. Genome Biol. Evol. 2017, 9, 1013-1029. [CrossRef] [PubMed]

44. Feller, A.; Machemer, K.; Braun, E.L.; Grotewold, E. Evolutionary and comparative analysis of MYB and bHLH plant transcription factors. Plant J. 2011, 66, 94-116. [CrossRef] [PubMed]

45. Lipsick, J.S. One billion years of Myb. Oncogene 1996, 13, 223-235. [PubMed]

46. Jiang, C.; Gu, J.; Chopra, S.; Gu, X.; Peterson, T. Ordered origin of the typical two- and three-repeat Myb genes. Gene 2004, 326, 13-22. [CrossRef]

47. Liu, C.; Xie, T.; Chen, C.; Luan, A.; Long, J.; Li, C.; Ding, Y.; He, Y. Genome-wide organization and expression profiling of the R2R3-MYB transcription factor family in pineapple (Ananas comosus). BMC Genom. 2017, 18, 503. [CrossRef]

48. Putra, E.T.S.; Zakaria, W.; Abdullah, N.A.P.; Saleh, G. Weak neck of Musa sp. cv. Rastali: A review on it's genetic, crop nutrition and post harvest. J. Agron. 2010, 9, 45-51. [CrossRef]

49. Droc, G.; Larivière, D.; Guignon, V.; Yahiaoui, N.; This, D.; Garsmeur, O.; Dereeper, A.; Hamelin, C.; Argout, X.; Dufayard, J.-F.; et al. The banana genome hub. Database 2013, 2013, bat035. [CrossRef]

50. Wang, X.; Wu, J.; Guan, M.; Zhao, C.-H.; Geng, P.; Zhao, Q. Arabidopsis MYB4 plays dual roles in flavonoid biosynthesis. Plant J. 2020, 101, 637-652. [CrossRef]

51. Zhou, M.; Wei, L.; Sun, Z.; Gao, L.; Meng, Y.; Tang, Y.; Wu, Y. Production and transcriptional regulation of proanthocyanidin biosynthesis in forage legumes. Appl. Microbiol. Biotechnol. 2015, 99, 3797-3806. [CrossRef] [PubMed] 
52. Gonzalez, A.; Mendenhall, J.; Huo, Y.; Lloyd, A. TTG1 complex MYBs, MYB5 and TT2, control outer seed coat differentiation. Dev. Biol. 2009, 325, 412-421. [CrossRef] [PubMed]

53. Deluc, L.; Barrieu, F.; Marchive, C.; Lauvergeat, V.; Decendit, A.; Richard, T.; Carde, J.-P.; Mérillon, J.-M.; Hamdi, S. Characterization of a grapevine R2R3-MYB transcription factor that regulates the phenylpropanoid pathway. Plant Physiol. 2006, 140, 499-511. [CrossRef] [PubMed]

54. Deluc, L.; Bogs, J.; Walker, A.R.; Ferrier, T.; Decendit, A.; Merillon, J.-M.; Robinson, S.P.; Barrieu, F. The transcription factor VvMYB5b contributes to the regulation of anthocyanin and proanthocyanidin biosynthesis in developing grape berries. Plant Physiol. 2008, 147, 2041-2053. [CrossRef]

55. Fan, Z.Q.; Ba, L.J.; Shan, W.; Xiao, Y.Y.; Lu, W.J.; Kuang, J.F.; Chen, J.Y. A banana R2R3-MYB transcription factor MaMYB3 is involved in fruit ripening through modulation of starch degradation by repressing starch degradation-related genes and MabHLH6. Plant J. 2018, 96, 1191-1205. [CrossRef]

56. Hartshorn, R. Some effects of acetylene on the ripening processes of bananas. Plant Physiol. 1931, 6, 467. [CrossRef]

57. Burg, S.P.; Burg, E.A. Molecular requirements for the biological activity of ethylene. Plant Physiol. 1967, 42, 144-152. [CrossRef]

58. Chen, L.; Zhong, H.; Kuang, J.; Li, J.; Lu, W.; Chen, J. Validation of reference genes for RT-qPCR studies of gene expression in banana fruit under different experimental conditions. Planta 2011, 234, 377-390. [CrossRef]

(C) 2020 by the authors. Licensee MDPI, Basel, Switzerland. This article is an open access article distributed under the terms and conditions of the Creative Commons Attribution (CC BY) license (http://creativecommons.org/licenses/by/4.0/). 